\title{
ON THE DERIVATION OF NECESSARY CONDITIONS FOR THE PROBLEM OF BOLZA*
}

\author{
BY G. A. BLISS AND I. J. SCHOENBERG
}

1. Introduction. The problem of Bolza with variable endpoints in the calculus of variations $\dagger$ is that of finding in a class of arcs

$$
y_{i}=y_{i}(x), \quad\left(i=1,2, \cdots, n ; x_{1} \leqq x \leqq x_{2}\right),
$$

satisfying the differential equations and end-conditions

$$
\begin{array}{lr}
\phi_{\alpha}\left(x, y, y^{\prime}\right)=0, & (\alpha=1,2, \cdots, m<n), \\
\psi_{\mu}\left[x_{1}, y\left(x_{1}\right), x_{2}, y\left(x_{2}\right)\right]=0, \quad(\mu=1,2, \cdots, p \leqq 2 n+2),
\end{array}
$$

one which minimizes an expression of the form

$$
I=G\left[x_{1}, y\left(x_{1}\right), x_{2}, y\left(x_{2}\right)\right]+\int_{x_{1}}^{x_{2}} f\left(x, y, y^{\prime}\right) d x .
$$

Morse and Myers $\ddagger$ have recently studied this problem in a somewhat different form. They seek to find in the class of arcs (1) satisfying the differential equations (2) and the end-conditions

$$
\begin{aligned}
x_{s}=x_{s}\left(\alpha_{1}, \cdots, \alpha_{r}\right), y_{i s}= & y_{i s}\left(\alpha_{1}, \cdots, \alpha_{r}\right), \\
& (s=1,2 ; i=1,2, \cdots, n),
\end{aligned}
$$

one which minimizes a sum

$$
I=\theta\left(\alpha_{1}, \cdots, \alpha_{r}\right)+\int_{x_{1}}^{x_{2}} f\left(x, y, y^{\prime}\right) d x .
$$

It has been customary to assume that the matrix of derivatives of the functions $\psi_{\mu}$ in (3) with respect to the variables $x_{s}$,

* Presented to the Society, October 29, 1932.

$\dagger$ †olza, Über den "Anormalen Fall" beim Lagrangeschen und Mayerschen Problem mit gemischten Bedingungen und variablen Endpunkten, Mathematische Annalen, vol. 74 (1913), pp. 430-446. Bliss, The problem of Bolza in the calculus of variations, Annals of Mathematics, (2), vol. 33 (1932), pp. 261-274. In the text here the latter paper will be referred to by the symbol $B_{2}$.

$\ddagger$ The problems of Lagrange and Mayer with variable end-points, Proceedings of the American Academy of Arts and Sciences, vol. 66 (1931), pp. 235-253. In the text this paper will be designated by the symbol $M M$. 
$y_{i s}=y_{i}\left(x_{s}\right),(s=1,2 ; i=1, \cdots, n)$, is of rank $p$ at the endvalues of the particular minimizing arc $E_{12}$ to be studied, ${ }^{*}$ an assumption which insures the non-singularity of the end-values and the independence of the conditions (3). For the parametric form (5) Morse and Myers do not assume that the matrix of derivatives of the functions (5) is of rank $r$ at the parameter values defining the ends of $E_{12}, \dagger$ and hence the problem as formulated by them seems to have greater generality. In a footnote on page 244 of their paper, Morse and Myers call attention to this fact and make the statement that the necessary conditions for a minimum deduced by them from the first variation can not easily be derived from the results given by Bliss for the corresponding Lagrange problem. $\ddagger$

In the following pages the authors wish to show how the methods used by Bliss for the Lagrange problem can be adapted with very slight modifications to deduce analogous theorems for the problem of Bolza. This will be done in $\$ 2$ below. By the introduction of new variables, in $\$ 3$, the parametric problem of Morse and Myers can be immediately reduced to one of the Bolza type. The necessary conditions usually derived from the first variation for the parametric form of the problem turn out to be simple corollaries of the corresponding theorems for the problem of Bolza.

The method used in deducing the theorem of $\$ 2$ still applies when the function $f$ in the expression (4) is identically zero. The problem is then that of Mayer with variable end-points as formulated by Bliss. § The theorem deduced by Myers\| for his modification of this problem is also an immediate consequence of the theorem of $\$ 2$ below by the method of $\$ 3$.

2. The Problem of Bolza. For a more precise formulation of the problem of Bolza see $B_{1}, \S \S 1$ and 8 , where the particular case

* Bolza, loc. cit., p. 433. Bliss, The problem of Lagrange in the calculus of variations, American Journal of Mathematics, vol. 52 (1930), condition (d) p. 690 . We shall refer to the latter paper in the text by the symbol $B_{1}$.

$\dagger M M$, pp. 236, footnote p. 244.

$\ddagger B_{1}, \S 8$, especially pp. 692,693 .

$\S$ The problem of Mayer with variable end-points, Transactions of this Society, vol. 19 (1918), pp. 305-314.

\| Adjoint systems in the problem of Mayer under general end conditions, this Bulletin, vol. 38 (1932), pp. 303-312, Theorem 4 of p. 311. 
$G=0$ is investigated, and $B_{2}, \S \S 1,2$. In particular we make the four assumptions $B_{1},(\mathrm{a})$, (b), (c), and (d). Furthermore $G\left(x_{1}, y_{i 1}, x_{2}, y_{i 2}\right)$ is supposed to be of class $C^{\prime}$ in a neighborhood of the end-points of $E_{12}$.

The additional term $G$ in the expression $I$ necessitates some slight changes in $B_{1}$, §8. Formula $B_{1}(45)$ becomes

$$
\begin{aligned}
\lambda_{0} I_{1}(\xi, \eta)= & -\int_{x_{1}}^{x_{2}} \lambda_{r} \xi_{r} d x-\lambda_{0} f\left(x_{1}\right) \xi_{1}-c_{i} \eta_{i}\left(x_{1}\right)+\lambda_{0} f\left(x_{2}\right) \xi_{2} \\
& +\eta_{i}\left(x_{2}\right) F_{y^{\prime}}\left(x_{2}\right)+\lambda_{0} G_{x_{1}} \xi_{1}+\lambda_{0} G_{y_{i 1}}\left[\eta_{i}\left(x_{1}\right)+y_{i 1}^{\prime} \xi_{1}\right] \\
& +\lambda_{0} G_{x_{2}} \xi_{2}+\lambda_{0} G_{y_{i 2}}\left[\eta_{i}\left(x_{2}\right)+y_{i 2}^{\prime} \xi_{2}\right] .
\end{aligned}
$$

The arguments following $B_{1}(45)$ hold without change and lead to the conclusion that there is a set of constants $\lambda_{0}, d_{1}, \cdots, d_{p}$, not all zero, such that the equation

$$
\begin{aligned}
0= & -\int_{x_{1}}^{x_{2}} \lambda_{r} \zeta_{r} d x+\left[\lambda_{0}\left(-f\left(x_{1}\right)+G_{x_{1}}+G_{y_{i 1}} y_{i 1}^{\prime}\right)\right. \\
& \left.+d_{\mu}\left(\psi_{\mu x_{1}}+\psi_{\mu y_{i 1}} y_{i 1}^{\prime}\right)\right] \xi_{1} \\
& +\left[\lambda_{0}\left(f\left(x_{2}\right)+G_{x_{2}}+G_{y_{i 2}} y_{i 2}^{\prime}\right)+d_{\mu}\left(\psi_{\mu x_{2}}+\psi_{\mu y_{i 2}} y_{i 2}^{\prime}\right)\right] \xi_{2} \\
& +\left[-c_{i}+\lambda_{0} G_{y_{i 1}}+d_{\mu} \psi_{\mu y_{i 1}}\right] \eta_{i}\left(x_{1}\right) \\
& +\left[F_{y^{\prime}}\left(x_{2}\right)+\lambda_{0} G_{y_{i 2}}+d_{\mu} \psi_{\mu y_{i 2}}\right] \eta_{i}\left(x_{2}\right)
\end{aligned}
$$

holds for every set of admissible variations $\xi_{1}, \xi_{2}, \eta_{i}(x)$ of the arc $E_{12}$. Choosing the constants $c_{i}$ so as to make the coefficients of $\eta_{i}\left(x_{1}\right)$ disappear, we infer, from the fact that $\zeta_{r}, \xi_{1}, \xi_{2}, \eta_{i}\left(x_{2}\right)$ can be chosen arbitrarily for admissible variations, that $\lambda_{r} \equiv 0$ and also that the matrix

$$
\begin{array}{cc}
-F\left(x_{1}\right)+\lambda_{0}\left(G_{x_{1}}+G_{y_{11}} y_{i 1}^{\prime}\right) & -F_{y_{i^{\prime}}}\left(x_{1}\right)+\lambda_{0} G_{y_{i_{1}}} \\
\psi_{\mu x_{1}}+\psi_{\mu y_{i 1}} y_{i 1}^{\prime} & \psi_{\mu y_{i_{1}}} \\
F\left(x_{2}\right)+\lambda_{0}\left(G_{x_{2}}+G_{y_{i_{2}}} y_{i_{2}}^{\prime}\right) & F_{y_{i^{\prime}}}\left(x_{2}\right)+\lambda_{0} G_{y_{i_{2}}} \\
\psi_{\mu x_{2}}+\psi_{\mu y_{i 2}} y_{i_{2}}^{\prime} & \psi_{\mu y_{i 2}}
\end{array}
$$

is of rank $p$. Hence also the matrix 


$$
\begin{array}{cc}
-F\left(x_{1}\right)+F_{y_{i^{\prime}}}\left(x_{1}\right) y_{i 1}^{\prime}+\lambda_{0} G_{x_{1}} & -F_{y_{i^{\prime}}}\left(x_{1}\right)+\lambda_{0} G_{y_{i 1}} \\
\psi_{\mu x_{1}} & \psi_{\mu y_{i 1}} \\
F\left(x_{2}\right)-F_{y_{i^{\prime}}}\left(x_{2}\right) y_{i 2}^{\prime}+\lambda_{0} G_{x_{2}} & F_{y_{i^{\prime}}}\left(x_{2}\right)+\lambda_{0} G_{y_{i 2}} \\
\psi_{\mu x_{2}} & \psi_{\mu y_{i 2}}
\end{array}
$$

is of rank $p$, which is equivalent to the statement that the relation

$$
\left[\left(F-F_{y_{i}} y_{i}^{\prime}\right) d x+F_{y_{i}} d y_{i}\right]_{1}^{2}+\lambda_{0} d G=0
$$

is a consequence of the system of equations

$$
\begin{array}{r}
d \psi_{\mu} \equiv \psi_{\mu x_{1}} d x_{1}+\psi_{\mu y_{i 1}} d y_{i 1}+\psi_{\mu x_{2}} d x_{2}+\psi_{\mu y_{i 2}} d y_{i 2}=0 \\
(\mu=1,2, \cdots, p) .
\end{array}
$$

The multipliers $\lambda_{0}, \lambda_{\alpha}(x)$ can not all vanish simultaneously at a point $x=a$, since this would imply $B_{i} \equiv 0$ in equation (17) of paper $B_{1}$, and also $v_{i}(a)=0$, from $B_{1}(16)$. Hence $B_{1}(17)$ would imply $v_{i} \equiv 0$; in particular $v_{i}\left(x_{1}\right)=c_{i}=0$, and $v_{i}\left(x_{2}\right)=F_{y^{\prime}}\left(x_{2}\right)=0$. From (7) we then find

$$
\begin{array}{ll}
d_{\mu}\left(\psi_{\mu x_{1}}+\psi_{\mu y_{i 1}} y_{i 1}^{\prime}\right)=0, & d_{\mu} \psi_{\mu y_{i 1}}=0 \\
d_{\mu}\left(\psi_{\mu x_{2}}+\psi_{\mu y_{i 2}} y_{i 2}^{\prime}\right)=0, & d_{\mu} \psi_{\mu y_{i 2}}=0
\end{array}
$$

from which we infer that $d_{\mu}=0$, since the rank of the matrix of the coefficients is $p$. Hence $\lambda_{0}=d_{\mu}=0$, in contradiction to our previous result. We have thus proved the following theorem.

THEOREM 1. For every minimizing arc $E_{12}$ for the problem of Bolza there exist constants $c_{i}$ and a function

$$
F=\lambda_{0} f+\lambda_{\alpha}(x) \phi_{\alpha}
$$

such that the equations

$$
F_{y_{i^{\prime}}}=\int_{x_{1}}^{x} F_{y_{i}} d x+c_{i}, \quad \phi_{\alpha}=0,
$$

hold at every point of $E_{12}$, and furthermore such that the end-points of $E_{12}$ satisfy, besides the equations (3), the condition that (8) holds for every set of differentials $d x_{1}, d y_{i 1}, d x_{2}, d y_{i 2}$ which satisfy the equations (9). The first multiplier $\lambda_{0}$ is a constant. The multipliers $\lambda_{\alpha}(x)$ have definite limits $\lambda_{\alpha}(x-0), \lambda_{\alpha}(x+0)$ at every value of $x$ 
on $x_{1} x_{2}$ and these limits are equal and the $\lambda_{\alpha}(x)$ are continuous except possibly at the values of $x$ defining the corners of $E_{12}$. The elements of the set $\lambda_{0}, \lambda_{\alpha}(x)$ do not vanish simultaneously at any point of $E_{12}$.

The form of the expression $I$ to be minimized has no bearing on questions regarding the normality of $E_{12}$; hence the results of $B_{1}, \S 9$ apply as well for the problem of Bolza.

3. The Problem of Bolza in the Formulation of Morse and Myers. In order to derive necessary conditions we suppose that a particular arc $E_{12}$ of class $D^{\prime}$, whose end-points are given by (5) for $(\alpha)=(0)$, minimizes $I$ in the class of all arcs (1) neighboring $E_{12}$ and satisfying the system (2) and whose end-points are given by (5) for values $(\alpha)$ near the values (0).

The problem of Morse and Myers can be phrased as a problem of Bolza as follows. We adjoin to the set of functions $y_{i}(x)$ new functions $\alpha_{h}(x)$ satisfying the differential equations $\alpha_{h}^{\prime}(x)=0$. The problem is then that of finding in the class of $\operatorname{arcs}$

$$
\begin{aligned}
& y_{i}=y_{i}(x), \quad \alpha_{h}=\alpha_{h}(x), \\
& \quad\left(i=1, \cdots, n ; h=1, \cdots, r ; x_{1} \leqq x \leqq x_{2}\right)
\end{aligned}
$$

satisfying the differential equations and end-conditions

$$
\phi_{\beta}\left(x, y, y^{\prime}\right)=0, \quad \alpha_{h}^{\prime}=0, \quad(\beta=1,2, \cdots, m),
$$

$\psi_{s}=x_{s}-x_{s}\left(\alpha_{1}, \cdots, \alpha_{r}\right)=0, \quad \psi_{i s}=y_{i s}-y_{i s}\left(\alpha_{1}, \cdots, \alpha_{r}\right)=0$,

one which minimizes the expression $I$ in (6). The particular minimizing arc $E_{12}{ }^{*}$ of the form (12), whose properties are to be studied, has $\alpha_{h}(x) \equiv 0,(h=1, \cdots, r)$. In the end-conditions (13) we think of the constants $\alpha_{h}$ as the initial values at $x=x_{1}$ of the functions $\alpha_{h}(x)$.

All of the assumptions of $\$ 2$ are satisfied by this problem in the Bolza form. In particular the functional matrix of the endconditions (13) has its determinant of derivatives with respect to the variables $x_{1}, x_{2}, y_{i 1}, y_{i 2}$ equal to unity. Applying the result of $\$ 2$ we know that there exist constants $c_{i}, c_{n+h}$ and a function

$$
F=\lambda_{0} f+\lambda_{\beta}(x) \phi_{\beta}+\lambda_{m+h}(x) \alpha_{h}^{\prime}
$$

such that the equations 


$$
F_{y_{i^{\prime}}}=\int_{x_{1}}^{x} F_{y_{i}} d x+c_{i}, \quad F_{\alpha_{h}^{\prime}}=\lambda_{m+h}=c_{n+h},
$$

hold along $E_{12}{ }^{*}$, and furthermore such that at the ends of $E_{12}{ }^{*}$ the equation

$$
\left[\left(F-y_{i}^{\prime} F_{\nu_{i^{\prime}}}\right) d x+F_{y_{i^{\prime}}} d y_{i}+\lambda_{m+h} d \alpha_{h}\right]_{1}^{2}+\lambda_{0} d \theta=0
$$

is a consequence of the equations

$$
d x_{s}-x_{s h} d \alpha_{h}=0, \quad d y_{i s}-y_{i s h} d \alpha_{h}=0,
$$

where $x_{s h}$ and $y_{i s h}$ are notations for $\partial x_{s} / \partial \alpha_{h}$ and $\partial y_{i s} / \partial \alpha_{h}$ at $(\alpha)$ $=(0)$. The constants $\lambda_{m+h}$ are all zero since in equation (14) they are coefficients of the differentials $d \alpha_{h 2}$ on which no conditions are imposed by the equations (15) which contain only $d \alpha_{h 1}=d \alpha_{h}$. We have therefore proved the following theorem.

THEOREM 2. With the minimizing arc $E_{12}$ there is associated a set of multipliers

$$
\lambda_{0}, \lambda_{\beta}(x), \quad(\beta=1,2, \cdots, m),
$$

and a set of constants $c_{1}, \cdots, c_{n}$ such that the equations

$$
F_{y_{i^{\prime}}}=\int_{x_{i}}^{x} F_{y_{i}} d x+c_{i}
$$

are satisfied at every point of $E_{12}$, where $F=\lambda_{0} f+\lambda_{\beta} \phi_{\beta}$. Furthermore the equation

$$
\left[\left(F-F_{y_{i}{ }^{\prime}} y_{i}^{\prime}\right) d x+F_{y_{i}{ }^{\prime}} d y_{i}\right]_{1}^{2}+\lambda_{0} d \theta=0
$$

is an identity in $d \alpha_{h}$ when $d x_{1}, d x_{2}, d y_{i 1}, d y_{i 2}$ and $d \theta$ are evaluated for $(\alpha)=(0)$ and expressed in terms of the differentials $d \alpha_{h}$ by the equations (15). The elements of (16) do not vanish simultaneously at any point of $E_{12}$.

According to the definitions of Morse and Myers, and Bliss, the admissible arc $E_{12}$ is normal $(A B)$ (see $M M, \S 5$ ) if and only if the arc $E_{12}{ }^{*}$ is normal for the corresponding problem of Bolza (see $\left.B_{1}, \S 9\right)$. Criteria for normality are given in $B_{1}, \S 9$, and $M M$, $\$ 5$. It can be shown now that Theorem 4 in $M M$ follows immediately out of the condition of Bliss concerning the nonvanishing of the determinant $B_{1}(49)$. From (13), $B_{1}(44)$, and 
the equations $\alpha_{h}^{\prime}=0$ we derive for a set of admissible variations along $E_{12}$ *

$$
\begin{aligned}
\Psi_{s}(\xi, \eta, u) & =\xi_{s}-x_{s h} u_{h}, \\
\Psi_{i s}(\xi, \eta, u) & =\eta_{i}\left(x_{s}\right)+y_{i s}^{\prime} \xi_{s}-y_{i s h} u_{h},
\end{aligned}
$$

where the constants $u_{h}$ are the variations of the constant functions $\alpha_{h}(x)$. From these we find immediately

$$
\Psi_{i s}=\eta_{i}\left(x_{s}\right)-\bar{\eta}_{i s}+y_{i s}^{\prime} \Psi_{s}
$$

with the notation

$$
\bar{\eta}_{i s}=\left(y_{i s h}-y_{i s}^{\prime} x_{s h}\right) u_{h}
$$

of Morse and Myers. A necessary and sufficient condition for $E_{12}{ }^{*}$ to be normal, according to the definition of Bliss, is that there exist $2 n+2$ sets of admissible variations $\xi_{s \mu}, \eta_{i \mu}(x), u_{h \mu}$, $(\mu=1, \cdots, 2 n+2)$, such that the determinant

$$
\left|\begin{array}{c}
\Psi_{s}\left(\xi_{\mu}, \eta_{\mu}, u_{\mu}\right) \\
\Psi_{i s}\left(\xi_{\mu}, \eta_{\mu}, u_{\mu}\right)
\end{array}\right|
$$

is different from zero. The first equations (17), and (18), show that this determinant is identical with the determinant $M M$ (5.1), with the notations $\xi_{s}$ in place of $\gamma_{s}$. It follows also that Theorem 5 in $M M$ is a corollary of the further results of Bliss in $B_{1}, \S 9$.

The University of Chicago 